Article

\title{
Fast Detection of 10 Cannabinoids by RP-HPLC-UV Method in Cannabis sativa L.
}

\author{
Mara Mandrioli ${ }^{1}$, Matilde Tura ${ }^{1}$, Stefano Scotti ${ }^{2}$ and Tullia Gallina Toschi ${ }^{1, * \mathbb{D}}$ \\ 1 Department of Agricultural and Food Sciences, Alma Mater Studiorum-University of Bologna, \\ Viale Fanin 40, 40127 Bologna, Italy; mara.mandrioli@unibo.it (M.M.); matilde.tura2@unibo.it (M.T.) \\ 2 Shimadzu Italia, Via G. B. Cassinis 7, 20139 Milano, Italy; sscotti@shimadzu.it \\ * Correspondence: tullia.gallinatoschi@unibo.it; Tel.: +39-051-209-6010
}

Academic Editor: Marcello Locatelli

Received: 10 May 2019; Accepted: 31 May 2019; Published: 4 June 2019

\begin{abstract}
Cannabis has regained much attention as a result of updated legislation authorizing many different uses and can be classified on the basis of the content of tetrahydrocannabinol (THC), a psychotropic substance for which there are legal limitations in many countries. For this purpose, accurate qualitative and quantitative determination is essential. The relationship between THC and cannabidiol (CBD) is also significant as the latter substance is endowed with many specific and non-psychoactive proprieties. For these reasons, it becomes increasingly important and urgent to utilize fast, easy, validated, and harmonized procedures for determination of cannabinoids. The procedure described herein allows rapid determination of 10 cannabinoids from the inflorescences of Cannabis sativa L. by extraction with organic solvents. Separation and subsequent detection are by RP-HPLC-UV. Quantification is performed by an external standard method through the construction of calibration curves using pure standard chromatographic reference compounds. The main cannabinoids dosed ( $\mathrm{g} / 100 \mathrm{~g}$ ) in actual samples were cannabidiolic acid (CBDA), CBD, and $\triangle 9$-THC (Sample L11 CBDA $0.88 \pm 0.04$, CBD $0.48 \pm 0.02, \Delta 9$-THC $0.06 \pm 0.00$; Sample L5 CBDA $0.93 \pm 0.06$, CBD $0.45 \pm 0.03, \triangle 9$-THC $0.06 \pm 0.00$ ). The present validated RP-HPLC-UV method allows determination of the main cannabinoids in Cannabis sativa $\mathrm{L}$. inflorescences and appropriate legal classification as hemp or drug-type.
\end{abstract}

Keywords: cannabinoids; Cannabis sativa L.; HPLC; validation

\section{Introduction}

Cannabis is classified into the family of Cannabaceae and initially encompassed three main species: Cannabis sativa, Cannabis indica, and Cannabis ruderalis [1]. Nowadays, Cannabis has only one species due to continuous crossbreeding of the three species to generate hybrids. In fact, all plants are categorized as belonging to Cannabis sativa and classified into chemotypes based on the concentration of the main cannabinoids. Depending on the THCA/CBDA ratio, some chemotypes have been distinguished. In particular, chemotype I or "drug-plants" have a TCHA/CBDA ratio >1.0, plants that exhibit an intermediate ratio are classified as chemotype II, chemotype III or "fiber-plants" have a THCA/CBDA ratio <1.0, plants that contain cannabigerolic acid (CBGA) as the main cannabinoid are classified as chemotype IV, and plants that contain almost no cannabinoids are classified as chemotype $\mathrm{V}$ [2-5].

Recently, in Italy the interest in Cannabis sativa L. has increased mainly due to the latest legislation (Legge n. 242 del 2 dicembre 2016) [6]. As a consequence, there is a request to develop cost-effective and easy-to-use quantitative and qualitative methods for analysis of cannabinoids.

The Italian regulatory framework has classified two types of Cannabis sativa L. depending on the content of $\triangle 9$-THC. In particular, fiber-type plants of Cannabis sativa L., also called "hemp", are 
characterized by a low content of $\Delta 9-\mathrm{THC}(<0.2 \% w / w)$. If the content of $\Delta 9-\mathrm{THC}$ is $>0.6 \% w / w$, it is considered as drug-type, also called "therapeutic" or "marijuana".

Industrial hemp is used in several sectors, such as in the pharmaceutical, cosmetic, food, and textile industries, as well as in energy production and building. In general, fiber-type plants are less used in the pharmaceutical field, where drug-type plants are more often employed [5]. However, there is also an increased interest in hemp varieties containing non-psychoactive compounds. In fact, the European Union has approved 69 varieties of Cannabis sativa L. for commercial use [7].

Hemp has a complex chemical composition that includes terpenoids, sugars, alkaloids, stilbenoids, quinones, and the characteristic compounds of this plant, namely cannabinoids. Cannabis sativa L. has several chemotypes, each of which is characterized by a different qualitative and quantitative chemical profile [5]. The cannabinoids, terpenes, and phenolic compounds in hemp are formed through secondary metabolism [3,8]. The term "cannabinoid" indicates terpenophenols derived from Cannabis. More than 90 cannabinoids are known, and some are derived from breakdown reactions [8]. Gaoni and Mechoulam [9] were the first to define cannabinoids "as a group of $C_{21}$ compounds typical of and present in Cannabis sativa, their carboxylic acids, analogs, and transformation products". Currently, cannabinoids have been classified according to their chemical structure, mainly seven types of cannabigerol (CBG); five types of cannabichromene ( $\mathrm{CBC}$ ); seven types of cannabidiol (CBD); the main psychoactive cannabinoid $\Delta 9$-tetrahydrocannabinol ( $\triangle 9$-THC) in nine different forms including its acid precursor ( $\Delta 9$-tetrahydrocannabinolic acid, $\Delta 9$-THCA); $\Delta 8$-tetrahydrocannabinol $(\Delta 8$-THC), which is a more stable isomer of $\triangle 9$-THC but $20 \%$ less active; three types of cannabicyclol (CBL); five different forms of cannabielsoin (CBE); seven types of Cannabinol (CBN), which is the oxidation artifact of $\triangle 9$-THC; cannabitriol (CBT); cannabivarin (CBDV); and tetrahydrocannabivarin (THCV) $[10,11]$. THC, CBD, CBG, CBN, and CBC are not biosynthesized in Cannabis sativa, and the plant produces the carboxylic acid forms of these cannabinoids (THCA, CBDA, CBGA, CBNA, and CBCA). Cannabinoid acids undergo a chemical decarboxylation reaction triggered by different factors, mainly temperature. This decarboxylation reaction leads to the formation of the respective neutral cannabinoids (THC, CBD, CBG, CBN, and CBC) $[12,13]$.

There are several methods to quantify cannabinoids [14-21], some of which require expensive mass spectrometry detectors [22-25]. Furthermore, there is a great deal of uncertainty around the use of gas chromatography (GC) for the titration of cannabinoids due to the high temperature of the injector and detector that can lead to the decarboxylation of cannabinoid acids if not derivatized correctly [26]. Moreover, recent studies have reported that cannabinoid acid decarboxylation is only partial, and as result the actual value is underestimated. An HPLC system allows for determination of the actual cannabinoid composition, both neutral and acid forms, without the necessity of the derivatization step [13].

It is necessary, in addition to honed methods, to develop new procedures with a view to discriminate different Cannabis varieties in order to identify and titrate cannabinoids in a simple way. These methods should ideally be fast, easy, robust, and cost-efficient as they can be used not only by research laboratories but also by small companies with a view on quality control.

This study focuses on the development, validation, and step-by-step explanation of a rapid and simple HPLC-UV method for identification and quantification of the main cannabinoids in hemp inflorescences that can be easily reproduced and applied. The method described is focused on the quantification of CBD but can also be applied to check the levels of THC.

\section{Results and Discussion}

\subsection{Method Development}

The aim of this work was to develop a new analytical method for determination of the main cannabinoids in hemp samples. In fact, the method described below can be used as a routine quality 
control procedure and can be applied by the pharmaceutical industry, small laboratories, or even small pharmacies.

A crucial aspect for accurate identification and quantification of analytes is optimization of separation conditions, and therefore various preliminary tests were carried out (e.g., mobile phase, detection wavelength). Different mobile phases were tested, and trials were performed with different compositions and gradient elution to optimize the separation of all 10 target compounds considered (File S2). The greatest difficulty was that of separating CBD and THCV, which in many cases co-eluted. It was also difficult to separate the isomers $\triangle 9$-THC and $\triangle 8$-THC. The best resolution of cannabinoids was obtained using a chromatographic column and, as an eluent mixture, water with $0.085 \%$ phosphoric acid and acetonitrile with $0.085 \%$ phosphoric acid.

The quantification of cannabinoids was made at $220 \mathrm{~nm}$ after testing different wavelengths (File S2). This wavelength represents the best compromise for all the cannabinoids considered and was selected to detect and integrate all compounds of interest within the dedicated concentration range. As far as chromatographic analysis is concerned, before using the instrument, the system was conditioned for $20 \mathrm{~min}$ by fluxing the eluent mixture in the instrument under the same conditions as the method, and then a chromatographic run was performed by injecting $5 \mu \mathrm{L}$ of acetonitrile to verify that the chromatographic system was adequately cleaned. Simultaneously with the analysis of the sample, standard solutions were injected at different concentrations for the construction of calibration curves and to evaluate the separation and identification of each compound. The identification of cannabinoids was performed by comparing their retention times with those obtained by the injection of pure standards and by an enhancing procedure. Figure 1 shows a chromatogram of a standard mixture of cannabinoids and Figure 2 shows a chromatogram of a sample of hemp.

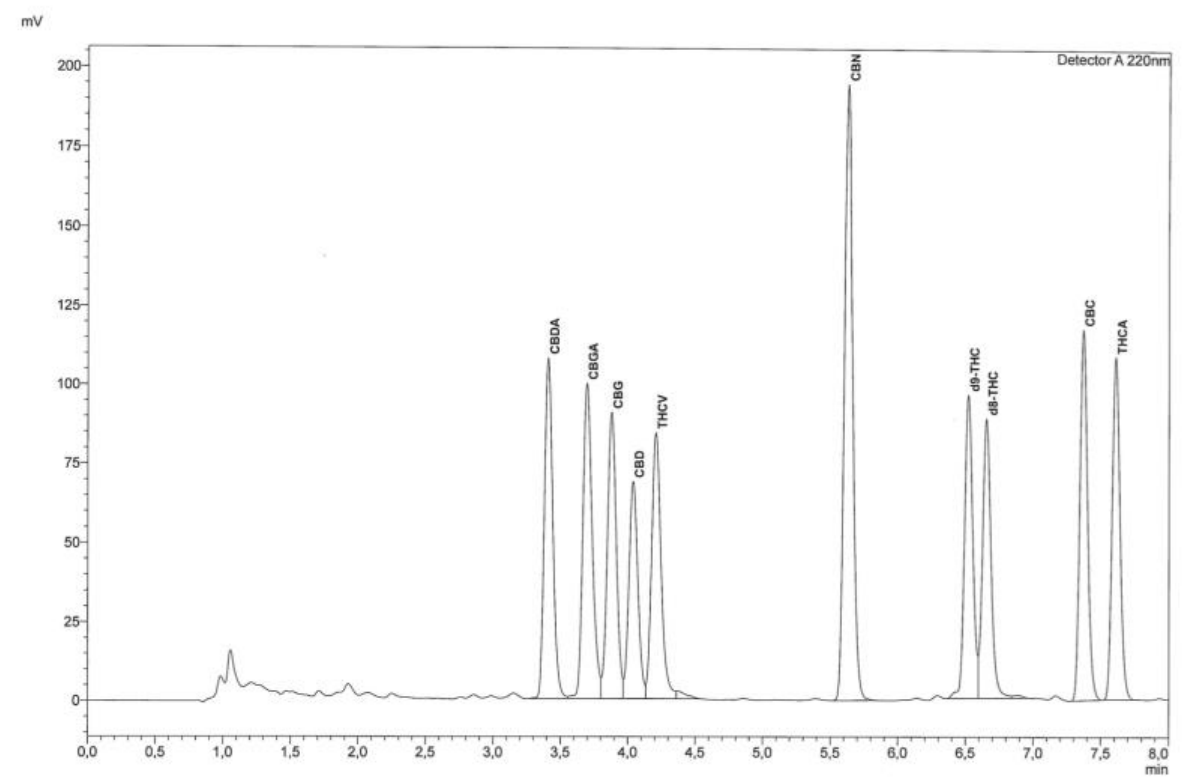

Figure 1. Chromatographic trace of a standard cannabinoid mixture analyzed by RP-HPLC-UV equipped with reverse phase C18 column.

Cannabinoids in different varieties of Cannabis sativa L. can be present in very different concentrations. In order to obtain good chromatographic separation and correct quantification, it may be necessary to dilute or concentrate the extract, performing two different injections. For example, in the case of high levels of CBDA or CBD it will be necessary to dilute the extract. For THC, it is often found at low concentration in hemp inflorescences, so it may be necessary to concentrate the extract before injection. In our case, $2 \mathrm{~mL}$ of filtered extract was dried using a weak nitrogen flow, and the dry extract was recovered in $500 \mu \mathrm{L}$ of acetonitrile. 




Figure 2. Chromatographic trace of Cannabis sativa L. inflorescence extract analyzed by RP-HPLC-UV equipped with a reverse phase C18 column.

\subsection{Validation}

\subsubsection{Precision}

The precision of the method was measured by the expression of repeatability $(r)$ and reproducibility $(R)$. Precision was expressed through coefficient of variation $(\mathrm{CV} \%)$.

\subsubsection{Repeatability, $\mathrm{R}$}

Table 1 shows data on the intraday and interday repeatability, evaluated as reported in Section 3.6, which demonstrates very high repeatability. In fact, the relative standard deviation (RSD) varied from 2.59 to 5.65 for intraday repeatability and from 2.83 to 5.05 for interday repeatability. In both cases, the highest RSD was found for CBDA, which is probably due its higher concentration compared to the other cannabinoids.

\subsubsection{Reproducibility, $\mathrm{R}$}

The RSDs obtained in the reproducibility studies are shown in Table 1. The maximum RSD value was 2.13 for CBGA. The other cannabinoids show RSD values lower than 1.91, and the lowest of the RSDs was 0.09 for CBDA, which is probably due to the higher concentration of this cannabinoid.

\subsubsection{Recovery}

The tests were performed by using three different concentrations to test the recovery values in the linearity range of the method.

Quantities of CBD $(4,8$, and $24 \mu \mathrm{g} / \mathrm{mL})$ were added, thus assessing concentrations similar to, higher, and lower than those found in samples.

Recovery was determined according to this modality for CBD and was $84.92 \%$.

An evaluation of recovery on all the compounds present in the sample was carried out by proceeding with a further extraction with $10 \mathrm{~mL}$ of methanol-chloroform on the sample residue after the usual extraction; in this extract, some cannabinoids were present, and indirectly the percentage of recovery was determined. 
Table 1. Validation parameters of RP-HPLC-UV method.

\begin{tabular}{|c|c|c|c|c|c|c|c|c|c|}
\hline Compound & $\mathbf{R}^{2}$ & $\begin{array}{c}{ }^{1} \text { LOD } \\
(\mu \mathrm{g} / \mathrm{mL})\end{array}$ & $\begin{array}{c}{ }^{2} \mathrm{LOQ} \\
(\mu \mathrm{g} / \mathrm{mL})\end{array}$ & $\begin{array}{c}{ }^{3} \text { LOD } \\
(\mu \mathrm{g} / \mathrm{mL})\end{array}$ & $\begin{array}{c}{ }^{4} \mathrm{LOQ} \\
(\mu \mathrm{g} / \mathrm{mL})\end{array}$ & $\begin{array}{c}\text { Intraday } \\
\text { (Repeatability) } \\
\text { RSD }\end{array}$ & $\begin{array}{c}\text { Interday } \\
\text { (Repeatability) } \\
\text { RSD }\end{array}$ & $\begin{array}{c}\text { Reproducibility } \\
\text { RSD }\end{array}$ & Recovery (\%) \\
\hline CBDA & 0.9999 & 0.34 & 1.05 & 0.11 & 0.37 & 5.65 & 5.05 & 0.09 & 96.06 \\
\hline CBGA & 0.9999 & 0.32 & 0.98 & 0.12 & 0.40 & 4.71 & 4.34 & 2.13 & 93.90 \\
\hline CBG & 0.9995 & 0.62 & 1.87 & 0.13 & 0.45 & 3.34 & 2.83 & 0.91 & 94.60 \\
\hline CBD & 0.9995 & 0.63 & 1.91 & 0.17 & 0.58 & 4.89 & 4.44 & 0.70 & 84.92 \\
\hline THCV & 0.9989 & 0.95 & 2.87 & 0.15 & 0.49 & - & - & N.d. * & N.d. * \\
\hline $\mathrm{CBN}$ & 0.9999 & 0.28 & 0.84 & 0.06 & 0.21 & 2.59 & 2.95 & 0.81 & 97.08 \\
\hline$\triangle 9-\mathrm{THC}$ & 0.9981 & 1.25 & 3.79 & 0.15 & 0.50 & 3.05 & 3.22 & 0.13 & 99.69 \\
\hline$\triangle 8$-THC & 0.9987 & 1.02 & 3.10 & 0.17 & 0.56 & 3.81 & 3.64 & 0.74 & 100 \\
\hline $\mathrm{CBC}$ & 0.9999 & 0.29 & 0.88 & 0.11 & 0.36 & 5.3 & 4.78 & 0.89 & 98.68 \\
\hline THCA & 0.9998 & 0.43 & 1.29 & 0.11 & 0.37 & 5.55 & 5.01 & 1.91 & 95.27 \\
\hline
\end{tabular}

${ }^{1}$ Limit of detection (LOD) determined by the calibration curves (Instrumental LOD $\left.=(3.3 \times \sigma) / \mathrm{m}\right) .{ }^{2}$ Limit of quantification (LOQ) determined by the calibration curves (Instrumental LOQ $=(10 \times \sigma) / \mathrm{m}) \cdot{ }^{3} \mathrm{LOD}$ determined by the signal-to-noise ratio (Instrumental LOD: $\mathrm{S} / \mathrm{N}=3$ ). ${ }^{4}$ LOQ determined by the signal-to-noise ratio (Instrumental LOQ: $\left.\mathrm{S} / \mathrm{N}=10\right) .{ }^{*}$ Not detectable. 
The percentage of recovery values, as shown in Table 1, were higher than $84.92 \%$ and can be considered very satisfactory. In fact, considering CBD, the percentages are higher than those previously reported in the literature [5].

\subsubsection{Detection Limit, LOD}

The instrumental limit of detection was determined by the calibration curve, according to the formulas expressed in Section 3.6. The instrumental limit of detection (LOD) values obtained for CBDA and CBGA (Table 1) were lower, while those of CBG and CBD were comparable with similar methods described in literature [5,27]. Low LOD values were found also for the other cannabinoids (THCV, CBN, $\triangle-9$ THC, $\triangle-8$ THC, CBC, THCA), indicating that the method is sensitive.

\subsubsection{Quantification Limit, LOQ}

The instrumental limit of quantification was determined by a calibration curve, according to the formulas expressed in Section 3.6, considering that the signal-to-noise method is particularly useful to quantify the cannabinoids present at lower concentrations, such as THC. As reported for the LODs, the instrumental limit of quantification (LOQ) values obtained for CBDA and CBGA (Table 1) were also lower than those reported in the literature, while those for CBG and CBD were comparable with those of other methods described for similar procedures [5,27]. In addition, the other cannabinoids (THCV, CBN, $\triangle-9$ THC, $\triangle-8$ THC, CBC, THCA) showed low LOQs. The instrumental noise was registered in $\mu \mathrm{V}$, by performing 3 blank injections with the ASTM method [28] given by the instrument, and a maximum $\mathrm{CV} \%$ of $3.49 \%$ was calculated for all individual compounds to determine the single LOD and LOQ, which was considered acceptable.

\subsubsection{Linearity}

In order to evaluate the linearity of the method, eight different points of standard mixture solutions were analyzed in triplicate by HPLC-UV.

The following equations are related to the calibration curves in a concentration range between $0.01-100 \mu \mathrm{g} / \mathrm{mL}$ : CBDA, $\mathrm{y}=18955 \mathrm{x}-1612.6\left(r^{2}=0.9999\right) ; \mathrm{CBGA}, \mathrm{y}=19796 \mathrm{x}-3475.7\left(r^{2}=0.9999\right)$; CBG, $\mathrm{y}=18094 \mathrm{x}-9195.3\left(r^{2}=0.9995\right) ; \mathrm{CBD}, \mathrm{y}=13703 \mathrm{x}-6009.5\left(r^{2}=0.9995\right) ; \mathrm{THCV}, \mathrm{y}=18534 \mathrm{x}-$ $15213\left(r^{2}=0.9989\right) ; \mathrm{CBN}, \mathrm{y}=34148 \mathrm{x}-7943.1\left(r^{2}=0.9999\right) ; \Delta 9-\mathrm{THC}, \mathrm{y}=19893 \mathrm{x}-31896\left(r^{2}=0.9981\right)$; $\triangle 8$-THC, $\mathrm{y}=17526 \mathrm{x}-18267\left(r^{2}=0.9987\right) ; \mathrm{CBC}, \mathrm{y}=18590 \mathrm{x}-4777.1\left(r^{2}=0.9999\right) ;$ THCA, $\mathrm{y}=18239 \mathrm{x}-$ $8969.3\left(r^{2}=0.9998\right)($ Table 1$)$.

With the aid of the equation obtained from the calibration curve, the quantity of each cannabinoid was calculated.

To express the data relative to the content of the individual cannabinoid as a percentage $(\%, \mathrm{p} / \mathrm{p})$ referred to the dried material, it is necessary to refer to the weight of the sample considering the dilution factor. The linearity in the concentration range analyzed was good for cannabinoid standards, being $r^{2}>0.998$, as reported before.

\subsection{Cannabinoids in Hemp Samples}

The method developed in this study was applied to quali-quantitative analysis of main cannabinoids in two samples of hemp inflorescences. The samples analyzed, belonging to the same variety of Cannabis sativa L., did not show a significant difference in the concentration of the target compounds. As shown in Table 2, CBDA is the only cannabinoid for which a different concentration was determined. The other cannabinoids had a similar or the same concentration (e.g., CBGA, CBG, CBN, $\Delta-9-\mathrm{THC}$, and $\Delta-8$-THC) in both samples. THCV was not found in the hemp inflorescence samples analyzed, as shown in Figure 2 and Table 2. $\Delta-9$-THC and $\Delta-8$-THC were found at a low concentration, below the legal limit. Under the current legislation regarding Cannabis sativa L. cultivation [6,29], in fact, the total content of THC must not be higher than $0.2 \%$ and in any case within $0.6 \%$. Indeed, only the hemp varieties reported in the Common catalogue of varieties of agricultural plant species can be 
cultivated without authorization [6,7]. These kinds of results confirmed that the analyzed samples were correctly classified as hemp, since the quantity of $\triangle 8$-THC and $\triangle 9$-THC was found to be lower than the limits established by the legislation. According to what is indicated in literature [30], in the hemp variety considered (Futura 75), the most present compound was CBDA, followed by CBD; all the other compounds were in very low amounts ranging from 0.01 to $0.06 \%$. CBGA is the compound from which all other cannabinoids are biosynthesized [5], which is probably why it was found at a low concentration in both samples examined.

The number of cannabinoids in hemp samples is reported in Table 2.

Table 2. Number of cannabinoids in hemp samples.

\begin{tabular}{|c|c|c|c|c|c|c|c|c|c|c|}
\hline \multicolumn{11}{|c|}{ Cannabinoids } \\
\hline Sample & $\begin{array}{c}\text { CBDA } \\
(\%)\end{array}$ & $\begin{array}{c}\text { CBGA } \\
(\%)\end{array}$ & $\begin{array}{c}\text { CBG } \\
(\%)\end{array}$ & $\begin{array}{l}\text { CBD } \\
(\%)\end{array}$ & $\begin{array}{c}\text { THCV } \\
(\%)\end{array}$ & $\begin{array}{c}\text { CBN } \\
(\%)\end{array}$ & $\begin{array}{c}\Delta 9-\mathrm{THC} \\
(\%)\end{array}$ & $\begin{array}{c}\Delta 8 \text {-THC } \\
(\%)\end{array}$ & $\begin{array}{c}\text { CBC } \\
(\%)\end{array}$ & $\begin{array}{c}\text { THCA } \\
(\%)\end{array}$ \\
\hline $\begin{array}{c}L 5 \\
C V \%\end{array}$ & $\begin{array}{c}0.93 \pm 0.06 \\
6.48\end{array}$ & $\begin{array}{c}0.02 \pm 0.00 \\
1.28\end{array}$ & $\begin{array}{c}0.02 \pm 0.00 \\
1.73\end{array}$ & $\begin{array}{c}0.45 \pm 0.03 \\
6.28\end{array}$ & N.d. ${ }^{*}$ & $\begin{array}{c}0.01 \pm 0.00 \\
1.49\end{array}$ & $\begin{array}{c}0.06 \pm 0.00 \\
0.21\end{array}$ & $\begin{array}{c}0.03 \pm 0.00 \\
2.20\end{array}$ & $\begin{array}{c}0.02 \pm 0.00 \\
2.98\end{array}$ & $\begin{array}{c}0.04 \pm 0.00 \\
7.17\end{array}$ \\
\hline
\end{tabular}

${ }^{*}$ Not detectable.

\section{Materials and Methods}

\subsection{Chemicals, Standards and Apparatus}

All chemicals used were of analytical grade. Methanol p.a CAS 67-56-1, chloroform p.a CAS 67-66-3, acetonitrile CAS 75-05-8, water CAS 7732-18-5, and orthophosphoric acid CAS 7664-38-2 were purchased from Sigma-Aldrich (St. Louis, MO, USA). Nitrogen, pure gas for analysis CAS 7727-37-9 was purchased from SIAD Spa (Bergamo, Italy). Standard mixture of phytocannabinoids $0.1 \%$ in acetonitrile: Cannabidiolic acid (0.01\%) CAS 1244-58-2, cannabigerolic acid (0.01\%) CAS 25555-57-1, cannabigerol (0.01\%) CAS 25654-31-3, cannabidiol (0.01\%) CAS 13956-29-1, tetrahydrocannabivarin (0.01\%) CAS 31262-37-0, cannabinol (0.01\%) CAS 521-35-7, tetrahydrocannabinolic acid (0.01\%) CAS 23978-85-0, $\Delta$-9-tetrahydrocannabinol (0.01\%) CAS 1972-08-3, $\Delta$-8-tetrahydrocannabinol (0.01\%) CAS 5957-75-5, cannabichromene (0.01\%) CAS Number 20675-51-8, were purchased from Cayman Chemical Company, (Ann Arbor, MI, USA). Cannabidiol $1.0 \mathrm{mg} / \mathrm{mL}$ in methanol CAS 13956-29-1: LGC Standards S.r.l., (Milan, Italy).

Analytical mill, IKA A11 Basic (IKA ${ }^{\circledR}$ Werke GMBH \& Co. KG, Germany). Analytical balance with precision of $0.1 \mathrm{mg}$, mod. E42, (Gibertini, Italy). Vortex vibrating shaker, mod. ST5, (Janke \& Kunkel, Germania). Centrifuge mod. ALC, PK 120 (Thermo Electron Corporation, Massachusetts, USA). Termoblock heating block, mod. A120, (Falc, Italy). Natural ventilation stove. Sieve with $1 \mathrm{~mm}$ meshes. Tilting shaker. Ultrasound bath Branson 2150, (Danbury-CT, USA). Volumetric flasks of 1, 2, 10 and $25 \mathrm{~mL}$. SOVIREL-type tubes with screw cap. Glass syringes with luer lock attachment, $0.45 \mu \mathrm{m}$ nylon membrane filters. Microsyringes from 1 to $1000 \mu \mathrm{L}$. HPLC Cannabis Analyzer for Potency Prominence-i LC-2030C equipped with a reverse phase C18 column, Nex-Leaf CBX Potency $150 \times 4.6 \mathrm{~mm}, 2.7 \mu \mathrm{m}$ with a guard column Nex-Leaf CBX $5 \times 4.6 \mathrm{~mm}, 2.7$, UV detector and acquisition software LabSolutions version 5.84 (Shimazu, Kyoto, Japan).

\subsection{Sampling}

The samples were supplied by a company that produces industrial hemp. In particular, two samples (L11 and L5) of inflorescences of Cannabis sativa L. Futura 75 were analyzed, having come from the same land and harvested in August 2017, and supplied by Enecta Srl. Sampling of material was carried out on a population of hemp plants, according to a systematic path, so that the sample taken was representative of the particle, excluding the edges, taking the upper third of the selected plant as indicated in Reg. (EU) No 1155/2017 [31]. The sample was dried in an oven at $35{ }^{\circ} \mathrm{C} \pm 1$ to constant weight, and gross wood parts and seeds with a length of more than $2 \mathrm{~mm}$ were removed. The samples 
were then subjected to grinding and subsequent sieving through a sieve with $1 \mathrm{~mm}$ meshes. The sieved material was transferred into polypropylene containers and stored under nitrogen atmosphere, protected from light at a temperature of $-20{ }^{\circ} \mathrm{C}$ until extraction. Three independent replicates were performed for each sample, and three HPLC injections were performed for each replication.

\subsection{Cannabinoid Extraction}

To extract cannabinoids, an aliquot of powder sample, about $25 \mathrm{mg}$, was weighed using an analytical balance; $10 \mathrm{~mL}$ of methanol-chloroform extraction solvent 9:1 $(v / v)$ was added as reported by De Backer et al. (2009) [32], Jin et al. (2017) [33], and was placed first for 10 min on an oscillating oscillator set at 350 oscillations per minute and then for $10 \mathrm{~min}$ in an ultrasonic bath. The sample was centrifuged for $10 \mathrm{~min}$ at $1125 \mathrm{~g}$, and the supernatant was removed. The extraction was performed twice. The two fractions containing cannabinoids were collected in a $25 \mathrm{~mL}$ volumetric flask and were brought to volume with methanol/chloroform $(9: 1, v / v)$. The samples were filtered with a $45 \mu \mathrm{m}$ nylon filter. Two $\mathrm{mL}$ of the filtered extract was transferred to a glass tube. The solvent was removed, leading to dryness with the help of a weak nitrogen flow, and recovered with $500 \mu \mathrm{L}$ acetonitrile. The solution was injected into an HPLC-UV.

\subsection{Preparation of Standard Solution}

Appropriate aliquots of a standard mixture of cannabinoids are diluted with acetonitrile to obtain solutions of known concentration, in particular eight points in a concentration range between 0.05 and $100 \mu \mathrm{g} / \mathrm{mL}(0.05,0.50,4.17,8.33,16.70,25.00,50.00,100.00 \mu \mathrm{g} / \mathrm{mL})$. The standard solutions were prepared to construct calibration curves for the 10 cannabinoids considered: CBDA, CBGA, CBG, CBD, THCV, CBN, $\triangle 9$-THC, $\triangle 8$-THC, CBC, and THCA. The standard solutions were stored away from light at a temperature of $-20{ }^{\circ} \mathrm{C}$. The stability of standard solutions stored at $-20{ }^{\circ} \mathrm{C}$ was evaluated every week for 3 months with the HPLC-UV system, and no degradation of cannabinoids was found.

\subsection{HPLC Conditions}

For the RP-HPLC analysis, the column was thermostated at $35^{\circ} \mathrm{C}$, and the autosampler was thermostated to $4{ }^{\circ} \mathrm{C}$. Sample concentration was $4 \mathrm{mg} / \mathrm{mL}$, and injection volume was $5.0 \mu \mathrm{L}$. UV detection was used at $220 \mathrm{~nm}$, and gradient elution was used at flow rate of $1.6 \mathrm{~mL} / \mathrm{min}$ according to the following procedure. Eluent mixture: Water $+0.085 \%$ phosphoric acid (A), acetonitrile $+0.085 \%$ phosphoric acid (B). Gradient elution: $70 \%$ of B up to $3 \mathrm{~min}, 85 \%$ of B to $7 \mathrm{~min}, 95 \%$ of B to 7.01 up to $8.00 \mathrm{~min}$, and $70 \%$ of B up to $10 \mathrm{~min}$. The eluent mixture was previously filtered with a Millipore system equipped with a $0.2 \mu \mathrm{m}$ nylon filter.

\subsection{Validation Parameters}

\subsubsection{Precision}

Precision is the closeness of agreement among independent test results, obtained with stipulated conditions and usually in terms of standard deviation or relative standard deviation [34].

Precision was calculated with the following formula: $C V \%=[(\operatorname{SD} / \bar{x}) \times 100]$, where $S D$ is the estimate of the standard deviation and $\bar{x}$ is the average of the replications made.

\subsubsection{Repeatability, $R$}

The repeatability (intraday) of the method was evaluated by analyzing three replicates of the same sample, injected three times on the same day, performed by the same operator with the same method and instrument. The result corresponds to the arithmetic mean of the three determinations made considering the estimate of the standard deviation (SD) calculated on the three replicates performed.

The repeatability (interday) of the method was evaluated by performing three replicates of the same sample, injected three times on three different days, performed by the same operator 
with the same method and instrument. The result corresponds to the arithmetic mean of the three determinations made considering the estimate of the standard deviation (SD) calculated on the three replicates performed.

\subsubsection{Reproducibility, $\mathrm{R}$}

Reproducibility was evaluated by the agreement between the results obtained on the same sample with the same procedure carried out by different operators in the laboratory and was measured with the coefficient of variation.

\subsubsection{Recovery}

Recovery is the fraction of analyte that was added to the sample being tested. Recovery was expressed as a percentage $(\mathrm{R}(\%))$ according to the following formula: $\mathrm{R}(\%)=[(\mathrm{Cf}-\mathrm{C}) / \mathrm{Cc}] \times 100$, where $\mathrm{Cf}$ is the endogenous amount of the cannabinoid in the sample plus the amount of standard added to the analyte under examination. $C$ is the endogenous amount present in the sample not added with the standard. Cc is the amount of the standard analyte added to the sample.

\subsubsection{Detection Limit, LOD}

The detection limit is the smallest amount or concentration of analyte in the sample that can be reliably distinguished from zero [34]. It can be calculated using the following formula: $\mathrm{LOD}=(3.3 \times$ $\sigma) / \mathrm{m}$, where: $\sigma$ represents the residual standard deviation of the calibration curve and $\mathrm{m}$ represents the slope of the calibration curve.

Furthermore, the LOD of the method from the signal $(\mathrm{S}) /$ noise $(\mathrm{N})$ ratio can be determined as LOD: $\mathrm{S} / \mathrm{N}=3$.

\subsubsection{Quantification limit, LOQ}

The quantification limit is the concentration of analyte below which it is determinable with a level of precision that is too low with inaccurate results. The LOQ can be determined according to the following formula: $\mathrm{LOQ}=(10 \times \sigma) / \mathrm{m}$, where $\sigma$ represents the residual standard deviation of the calibration curve and $\mathrm{m}$ represents the slope of the calibration curve.

The LOQ of the method can also be determined by the signal-to-noise ratio $(\mathrm{S} / \mathrm{N})$ : LOQ: $\mathrm{S} / \mathrm{N}=10$.

\subsubsection{Linearity}

Linearity can be tested by examination of a plot of residuals produced by linear regression of the responses on the concentrations in an appropriate calibration set [34].

In order to quantify the analytes of interest, the equation of the calibration curve obtained for each standard is used. The equation is: $y=a x+b$, where $y=$ area of the analyte obtained by HPLC/UV analysis, $\mathrm{a}=$ slope of the calibration curve, $\mathrm{x}=$ unknown concentration $(\mu \mathrm{g} / \mathrm{mL})$ of analyte in the sample, $b=$ intercept of the calibration curve.

\section{Conclusions}

One of the most relevant problems in analytical determinations for quality control, especially when there are legal problems related with quantitation, such as for cannabis, relates to the proficiency of laboratories. Therefore, detailed and validated procedures that are freely available are essential for the full understanding of any analytical step and its careful application. This is also true for "daily" methods that can be easily applied for quality control, carried out using traditional RP-HPLC and UV-Vis detectors, with less efficient performance than diode-array detectors but with lower costs, rendering them affordable even for small laboratories.

The validated method described herein allows the quantitative determination of the 10 most relevant cannabinoids using a single wavelength $(220 \mathrm{~nm})$ in $8 \mathrm{~min}$. A full separation is obtained, even 
in the elution sequence of a difficult resolution, of the group of peaks related to CBGA, CBG, CBD, and THCV (from 3.5 to $4.5 \mathrm{~min}$ ).

The method is applied to cannabis inflorescences and involves extraction in methanol/ chloroform, drying of the extract, taking it up in acetonitrile and injection into an HPLC. The method has sensitivity and accuracy to discriminate samples with amounts of $\Delta-9$ - and $\Delta-8$-THC (total THC content) that are below the limit of $0.2 \%$ from those that are subjected to legal restrictions in many EU countries, with a total THC content above $0.6 \%$, which cannot be classified as hemp. Due to its simplicity and rapidity, it can be used to check raw material or crops during the harvesting period.

A detailed standard operating procedure (SOP), as a supplementary information file, is also available, so that any operator with basic knowledge of HPLC can easily apply it and make all the elution and calibration control checks using commercially available mixtures of standards, which are more affordable and sustainable than single cannabinoid standards in terms of costs and solvents used for calibration.

Supplementary Materials: The following are available online. File S1: Standard operating procedure (SOP) of the method presented in this article, Table S1: Calibration curves relating to the standard solution of 10 cannabinoids determined by RP-HPLC-UV method, Figure S1: Calibration curves relating to the standard solution of 10 cannabinoids determined by RP-HPLC-UV method, File S2: Preliminary tests carried out for development of the analytical procedure by RP-HPLC-UV.

Author Contributions: Conceptualization, T.G.T., M.M. and M.T.; Methodology, M.M.; Software, M.M. and S.S.; Validation, M.M.; Formal analysis, M.M.; Investigation, T.G.T. and M.M.; Resources, T.G.T. and M.M.; Data curation, M.M., T.G.T. and M.T.; Writing-original draft preparation, M.M., M.T. and T.G.T.; writing-review and editing, T.G.T. and S.S.; Visualization, T.G.T.; Supervision, T.G.T.; project administration, T.G.T.; funding acquisition, T.G.T.

Funding: This research received no external funding; this trial received financial support from Enecta Srl.

Acknowledgments: The authors gratefully acknowledge Enecta Srl for providing samples. The experimentation was conducted in the context of a PhD project entitled "Harmonized procedures of analysis of medical, herbal, food and industrial cannabis: development and validation of cannabinoids' quality control methods, of extraction and preparation of derivatives from the plant raw material, according to the product destination" and funded by ENECTA Srl.

Conflicts of Interest: The authors declare no conflict of interest.

\section{References}

1. Montserrat-de la Paz, S.; Marín-Aguilar, F.; García-Gimenez, M.D.; Fernández-Arche, M.A. Hemp (Cannabis sativa L.) seed oil: Analytical and phytochemical characterization of the unsaponifiable fraction. J. Agric. Food Chem. 2014, 62, 1105-1110. [CrossRef] [PubMed]

2. Appendino, G.; Chianese, G.; Taglialatela-Scafati, O. Cannabinoids: Occurrence and medicinal chemistry. Curr. Med. Chem. 2011, 18, 1085-1099. [CrossRef]

3. Andre, C.M.; Hausman, J.F.; Guerriero, G. Cannabis sativa: The plant of the thousand and one molecules. Front. Plant. Sci. 2016, 7, 19. [CrossRef] [PubMed]

4. Aizpurua-Olaizola, O.; Soydaner, U.; Öztürk, E.; Schibano, D.; Simsir, Y.; Navarro, P.; Usobiaga, A. Evolution of the cannabinoid and terpene content during the growth of Cannabis sativa plants from different chemotypes. J. Nat. Prod. 2016, 79, 324-331. [CrossRef]

5. Brighenti, V.; Pellati, F.; Steinbach, M.; Maran, D.; Benvenuti, S. Development of a new extraction technique and HPLC method for the analysis of non-psychoactive cannabinoids in fibre-type Cannabis sativa L.(hemp). J. Pharm. Biomed. Anal. 2017, 143, 228-236. [CrossRef] [PubMed]

6. Legge 2 Dicembre 2016, n.242. Disposizioni per la Promozione della Coltivazione e della Filiera Agroindustriale della Canapa (16G00258), GU Serie Generale n. 304 del 30-12-2016. Available online: https://www.gazzettaufficiale.it/eli/id/2016/12/30/16G00258/sg (accessed on 1 June 2019).

7. European Commission (EC). European Union Common Catalogue of Varieties of Agricultural Plant Species, Plant Variety Database. Available online: http://ec.europa.eu/food/plant/plant_propagation_material/plant_ variety_catalogues_databases/search//public/index.cfm (accessed on 1 June 2019). 
8. Pisanti, S.; Malfitano, A.M.; Ciaglia, E.; Lamberti, A.; Ranieri, R.; Cuomo, G.; Laezza, C. Cannabidiol: State of the art and new challenges for therapeutic applications. Pharmacol. Ther. 2017, 175, 133-150. [CrossRef] [PubMed]

9. Mechoulam, R.; Gaoni, Y. Recent advances in the chemistry of hashish. In The Chemistry of Organic Natural Products/Progrès dans la Chimie des Substances Organiques Naturelles; Springer International Publishing: Vienna, Austria, 1967; pp. 175-213.

10. Radwan, M.M.; Wanas, A.S.; Chandra, S.; ElSohly, M.A. Natural Cannabinoids of Cannabis and Methods of Analysis. In Cannabis sativa L.-Botany and Biotechnology, 1st ed.; Springer International Publishing AG: Cham, Switzerland, 2017; pp. 161-182.

11. Leghissa, A.; Hildenbrand, Z.L.; Schug, K.A. A review of methods for the chemical characterization of cannabis natural products. J. Sep. Sci 2018, 41, 398-415. [CrossRef] [PubMed]

12. Citti, C.; Pacchetti, B.; Vandelli, M.A.; Forni, F.; Cannazza, G. Analysis of cannabinoids in commercial hemp seed oil and decarboxylation kinetics studies of cannabidiolic acid (CBDA). J. Pharm Biomed. Anal. 2018, 149, 532-540. [CrossRef] [PubMed]

13. Citti, C.; Braghiroli, D.; Vandelli, M.A.; Cannazza, G. Pharmaceutical and biomedical analysis of cannabinoids: A critical review. J. Pharm Biomed. Anal. 2018, 147, 565-579. [CrossRef] [PubMed]

14. Rodrigues, A.; Yegles, M.; Van Elsué, N.; Schneider, S. Determination of cannabinoids in hair of CBD rich extracts consumers using gas chromatography with tandem mass spectrometry (GC/MS-MS). Forensic. Sci. Int. 2018, 292, 163-166. [CrossRef]

15. Cardenia, V.; Gallina Toschi, T.; Scappini, S.; Rubino, R.C.; Rodriguez Estrada, M.T. Development and validation of a Fast gas chromatography/mass spectrometry method for the determination of cannabinoids in Cannabis sativa L. J. Food Drug Anal. 2018, 26, 1283-1292. [CrossRef] [PubMed]

16. Leghissa, A.; Hildenbrand, Z.L.; Foss, F.W.; Schug, K.A. Determination of cannabinoids from a surrogate hops matrix using multiple reaction monitoring gas chromatography with triple quadrupole mass spectrometry. $J$. Sep. Sci 2018, 41, 459-468. [CrossRef] [PubMed]

17. Patel, B.; Wene, D.; Fan, Z.T. Qualitative and quantitative measurement of cannabinoids in cannabis using modified HPLC/DAD method. J. Pharm Biomed. Anal. 2017, 146, 15-23. [CrossRef] [PubMed]

18. Burnier, C.; Esseiva, P.; Roussel, C. Quantification of THC in Cannabis plants by fast-HPLC-DAD: A promising method for routine analyses. Talanta 2019, 192, 135-141. [CrossRef] [PubMed]

19. Pellati, F.; Brighenti, V.; Sperlea, J.; Marchetti, L.; Bertelli, D.; Benvenuti, S. New Methods for the Comprehensive Analysis of Bioactive Compounds in Cannabis sativa L.(hemp). Molecules 2018, 23, 2639. [CrossRef]

20. Ciolino, L.A.; Ranieri, T.L.; Taylor, A.M. Commercial cannabis consumer products part 2: HPLC-DAD quantitative analysis of cannabis cannabinoids. Forensic. Sci. Int. 2018, 289, 438-447. [CrossRef]

21. Fekete, S.; Sadat-Noorbakhsh, V.; Schelling, C.; Molnár, I.; Guillarme, D.; Rudaz, S.; Veuthey, J.L. Implementation of a generic liquid chromatographic method development workflow: Application to the analysis of phytocannabinoids and Cannabis sativa extracts. J. Pharm. Biomed. Anal. 2018, 155, 116-124. [CrossRef]

22. Purschke, K.; Heinl, S.; Lerch, O.; Erdmann, F.; Veit, F. Development and validation of an automated liquid-liquid extraction GC/MS method for the determination of THC, 11-OH-THC, and free THC-carboxylic acid (THC-COOH) from blood serum. Anal. Bioanal. Chem. 2016, 408, 4379-4388. [CrossRef]

23. Pacifici, R.; Marchei, E.; Salvatore, F.; Guandalini, L.; Busardò, F.P.; Pichini, S. Evaluation of cannabinoids concentration and stability in standardized preparations of cannabis tea and cannabis oil by ultra-high performance liquid chromatography tandem mass spectrometry. Clin. Chem. Lab. Med. 2017, 55, 1555-1563. [CrossRef]

24. Casiraghi, A.; Roda, G.; Casagni, E.; Cristina, C.; Musazzi, U.M.; Franzè, S.; Gambaro, V. Extraction Method and Analysis of Cannabinoids in Cannabis Olive Oil Preparations. Planta Med. 2018, 84, 242-249. [CrossRef]

25. Lin, S.Y.; Lee, H.H.; Lee, J.F.; Chen, B.H. Urine specimen validity test for drug abuse testing in workplace and court settings. J. Food Drug Anal. 2018, 26, 380-384. [CrossRef] [PubMed]

26. Mudge, E.M.; Murch, S.J.; Brown, P.N. Leaner and greener analysis of cannabinoids. Anal. Bioanal. Chem. 2017, 409, 3153-3163. [CrossRef] [PubMed] 
27. Gul, W.; Gul, S.W.; Radwan, M.M.; Wanas, A.S.; Mehmedic, Z.; Khan, I.I.; ElSohly, M.A. Determination of 11 cannabinoids in biomass and extracts of different varieties of Cannabis using high-performance liquid chromatography. J. AOAC Int. 2015, 98, 1523-1528. [CrossRef] [PubMed]

28. ASTM. ASTM E685-93(2013), Standard Practice for Testing Fixed-Wavelength Photometric Detectors Used in Liquid Chromatography; ASTM International: West Conshohocken, PA, USA, 2013. Available online: www.astm.org (accessed on 1 June 2019).

29. Regulation (EU) No 1307/2013 of the European Parliament and of the Council of 17 December 2013 Establishing Rules for Direct Payments to Farmers under the Support Schemes within the Framework of the Common Agricultural Policy and Repealing Council Regulation (EC) No 637/2008 and Council Regulation (EC) No 73/2009. Available online: https://eur-lex.europa.eu/legal-content/EN/TXT/?uri=celex:32013R1307 (accessed on 1 June 2019).

30. del M. Contreras, M.; Jurado-Campos, N.; Sánchez-Carnerero Callado, C.; Arroyo-Manzanares, N.; Fernández, L.; Casano, S.; Ferreiro-Vera, C. Thermal desorption-ion mobility spectrometry: A rapid sensor for the detection of cannabinoids and discrimination of Cannabis sativa L. chemotypes. Sens. Actuators B Chem. 2018, 273, 1413-1424. [CrossRef]

31. Commission Delegated Regulation (EU) 2017/1155 of 15 February 2017 Amending Delegated Regulation (EU) No 639/2014 as Regards the Control Measures Relating to the Cultivation of Hemp, Certain Provisions on the Greening Payment, the Payment for Young Farmers in Control of a Legal Person, the Calculation of the per Unit Amount in the Framework of Voluntary Coupled Support, the Fractions of Payment Entitlements and Certain Notification Requirements Relating to the Single Area Payment Scheme and the Voluntary Coupled Support, and Amending Annex X to Regulation (EU) No 1307/2013 of the European Parliament and of the Council. Available online: https:/eur-lex.europa.eu/eli/reg_del/2017/1155/oj (accessed on 1 June 2019).

32. De Backer, D.B.; Debrus, B.; Lebrun, P. Innovative development and validation of an HPLC/DAD method for the qualitative and quantitative determination of major cannabinoids in cannabis plant material. $J$. Chromatogr. B Anal. Technol. Biomed. Life Sci. 2009, 877, 4115-4124. [CrossRef] [PubMed]

33. Jin, D.; Jin, S.; Yu, Y.; Lee, C.; Chen, J. Analytical \& Bioanalytical Techniques Classification of Cannabis Cultivars Marketed in Canada for Medical Purposes by Quantification of Cannabinoids and Terpenes Using HPLC-DAD and GC-MS. J. Anal. Bioanal. Tech. 2017, 8, 1-9.

34. Thompson, M.; Ellison, S.L.; Wood, R. Harmonized guidelines for single-laboratory validation of methods of analysis (IUPAC Technical Report). Pure Appl. Chem. 2002, 74, 835-855. [CrossRef]

Sample Availability: Samples of fiber-type hemp extracts are available from the authors.

(C) 2019 by the authors. Licensee MDPI, Basel, Switzerland. This article is an open access article distributed under the terms and conditions of the Creative Commons Attribution (CC BY) license (http://creativecommons.org/licenses/by/4.0/). 